\title{
Delfines de río: especies indicadoras del estado de salud de los ecosistemas acuáticos de la Amazonia y la Orinoquia
}

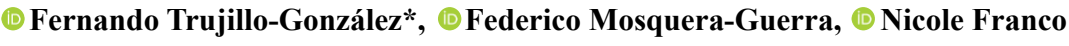 \\ Fundación Omacha
}

Artículo de posesión para el ingreso como miembro correspondiente a la

Academia Colombiana de Ciencias Exactas, Físicas y Naturales el 22 de abril del 2019

\section{Resumen}

En el presente estudio se emplearon los tamaños poblacionales de dos géneros de delfines de río (Inia y Sotalia) como indicadores del estado de salud de 23 ecosistemas acuáticos asociados con las cuencas de los ríos Amazonas y Orinoco. La información se recopiló durante 30 expediciones científicas realizadas entre el 2006 y el 2017 en Colombia, Ecuador, Perú, Bolivia, Brasil y Venezuela. Los resultados evidenciaron procesos de degradación ecosistémica en los ríos Caquetá, Putumayo, Iténez y Grande en la cuenca del Amazonas, y en el TocantinsAraguaia, el Guaviare y la cuenca alta del Orinoco. Esta condición puede verse incrementada en las próximas décadas debido a la pérdida de conectividad entre los Andes y el Amazonas por la presencia de 142 hidroeléctricas ya existentes y 160 que están en fase de planeación y han sido propuestas para los ríos que drenan desde las cabeceras andinas al Amazonas. Los impactos ecosistémicos en las redes tróficas acuáticas de estos sistemas generados por este tipo de infraestructura se han estudiado poco. Los resultados del estudio aportan importantes elementos para entender los procesos de degradación ocasionados por la fragmentación y la pérdida de la conectividad fluvial en la Amazonia y la Orinoquia a partir de los tamaños poblacionales de los delfines de río. Estos cetáceos tienen variados requerimientos ecológicos y se han adaptado evolutivamente a los cambios estacionales predecibles. Estas características ecológicas convierten a los delfines de río en excelentes indicadores para entender las afectaciones en los pulsos de inundación y sus efectos sobre la funcionalidad ecosistémica de los complejos sistemas hidrográficos de las cuencas del Amazonas y el Orinoco. (C) 2019. Acad. Colomb. Cienc. Ex. Fis. Nat.

Palabras clave: Bioindicación; Conservación; Cuenca; Suramérica; Tamaños poblacionales.

River dolphins: Species that indicate the state of health of the aquatic ecosystems of the Amazon and Orinoco regions

\begin{abstract}
In the present study, we used the population sizes of river dolphins (Inia and Sotalia) as indicators of the state of health of 20 aquatic ecosystems associated with the basins of the Amazon and Orinoco Rivers. The information was collected during 30 scientific expeditions carried out during 2006 and 2017 in Colombia, Ecuador, Perú, Bolivia, Brazil, and Venezuela. The results show intense processes of ecosystem degradation in the Caquetá, Putumayo, Iténez, and Grande Rivers in the Amazon basin and the Tocantins-Araguaia and Guaviare Rivers, as well as in the upper basin of the Orinoco River. This condition may be increased in the coming decades due to the loss of connectivity between the Andes and the Amazon due to the presence of 142 already existing hydroelectric dams and other 160 which are in the planning stage on the rivers running from the Andean headwaters to the Amazon. The ecosystemic impacts of this type of infrastructure on the aquatic trophic networks of these systems have been poorly studied. Our results provide important elements for understanding the degradation processes caused by the fragmentation and the loss of fluvial connectivity in the Amazon and Orinoco regions using the population sizes of river dolphins as indicators. These cetaceans have extensive ecological requirements and are evolutionarily adapted to predictable seasonal changes. These ecological characteristics make river dolphins excellent indicators to understand the effects on flood pulses and their effects on the ecosystem functionality of the complex hydrographic systems of the Amazon and Orinoco basins. (C) 2019. Acad. Colomb. Cienc. Ex. Fis. Nat.
\end{abstract}

Key words: Bioindication; Conservation; Basin; South America; Population sizes.

\footnotetext{
*Correspondencia:

Fernando Trujillo-González, fernando@omacha.org; Recibido: 28 de septiembre de 2018; Aceptado: 28 de febrero de 2019; Editor: Jaime Cantera
} 


\section{Introducción}

Los delfines de río se encuentran entre los mamíferos acuáticos más amenazados del planeta y están clasificados en la categoría "En peligro" (EN) (da Silva, et al., 2018). Su distribución geográfica abarca países de Asia y Suramérica donde las actividades antrópicas han deteriorado de manera preocupante los ecosistemas acuáticos donde habitan (Reeves \& Leatherwood, 1994; Smith \& Braulik, 2008; Trujillo, et al., 2010; Mosquera-Guerra, et al., 2015). En Asia se reportan delfines de agua dulce en el río Yangtze (China) y en el complejo hidrográfico del río Ganges (India, Nepal), en el Indo (Pakistán) y en el Megna (Bangladesh) (Smith \& Braulik, 2008). En el caso de China, en el 2005 se reportó la extinción de la especie Lipotes vexillifer (Turvey, et al., 2007), siendo una de las más recientes desapariciones de un gran mamífero en tiempos modernos. En Suramérica existen al menos cuatro especies de delfines de río: 1) el bufeo o tonina (Inia geoffrensis Blainville, 1817) con las subespecies: I. g. geoffrensis (Blainville, 1817) en la cuenca del Amazonas e I. g. humboldtiana (Pilleri and Gihr, 1977) en la cuenca del Orinoco; 2) el bufeo boliviano (Inia boliviensis D'Orbigny, 1834) presente en los ríos Mamoré, Itenez, Grande y en la cuenca alta de río Madeira en Bolivia y Brasil; 3) el boto de Araguaia (Inia araguaiaensis, Hrbek, et al., 2014) en la cuenca de los ríos Tocantins y Araguaia, y 4) el tucuxi (Sotalia fluviatilis Gervais \& Deville, 1853) en la Amazonia (Caballero, 2007; Shostell \& RuízGarcía, 2010; Hrbek, et al., 2014; Gravena, et al., 2015; Mosquera-Guerra, et al., 2015a). Es importante mencionar que la resolución taxonómica para los géneros Inia y Sotalia aún se encuentra en revisión, y es posible que en un futuro se logre la descripción de nuevas especies.

Las principales amenazas contra la conservación de los delfines de río en Asia y Suramérica se concentran en la pérdida, el deterioro y la fragmentación de sus hábitats ocasionados por los intensos procesos de deforestación, la construcción de hidroeléctricas y la contaminación de ríos con metales pesados como el mercurio, con pesticidas e hidrocarburos (Trujillo, et al., 2010; Pavanato, et al., 2016; Mosquera-Guerra, et al., 2015d; 2018; 2018a). A ello se añade el colapso de los recursos pesqueros que trae como consecuencia la reducción en la disponibilidad de presas para estos cetáceos, así como las interacciones biológicas y operacionales con las pesquerías comerciales y artesanales que ocasionan percepciones negativas y conflictos con los delfines de río (Trujillo, et al., 2010a; Mosquera-Guerra, et al., 2015b).

En consecuencia, durante las últimas décadas se recomendó el diseño de metodologías apropiadas para la estimación poblacional de los delfines de río, con el fin de establecer si realmente sus poblaciones se encontraban estables o disminuían (Perrin, et al., 1989, Reeves, et al., 1994a, International Whaling Commission - IWC, 2000). Entre 1980 y 1990 solo existían este tipo de programas de investigación con carácter permanente en países como Colombia y Brasil, y los esfuerzos de trabajo aportaban información importante, pero en una escala geográfica limitada. Por esa razón, a partir del 2001 se promovió desde Colombia la consolidación de un programa de estimación de la abundancia de delfines de río. El objetivo de esta iniciativa fue el de estandarizar el proceso metodológico para la estimación de la población de estos cetáceos de manera que fuera replicable, y que la información recolectada en diferentes localidades fuera comparable. A lo largo de estos años se han realizado 30 expediciones científicas en seis países, con lo cual se ha logrado incrementar de manera significativa el conocimiento acerca de los tamaños poblacionales de los delfines de río en Suramérica (Gómez, et al., 2011; Williams, et al., 2016). Esta información fue fundamental para la construcción de los rangos poblacionales como criterio para la determinación del estado de las condiciones ecológicas de los ecosistemas acuáticos evaluados en las cuencas del Amazonas, el Orinoco y el Tocantins.

\section{Materiales y métodos}

Area de estudio. Se hicieron 30 estimaciones poblacionales de delfines de río en ecosistemas acuáticos asociados con las cuencas del Amazonas, el Orinoco y el Tocantins en Colombia, Ecuador, Perú, Bolivia, Brasil y Venezuela, entre los años 2006 y 2018. Los sistemas acuáticos evaluados en la cuenca del Amazonas fueron los ríos Amazonas, Putumayo, Napo, Marañón, Ucayali, Cuyabeno, Yasuní, Lagartococha, Mamoré, Iténez-Guaporé, Grande, Madeira, Javari, Loretoyacu, Caquetá-Japurá, Purús, y Tapajos; en la cuenca del Orinoco se evaluaron los ríos Orinoco (Casiquiare, Medio y Delta), Meta, Guaviare, Bita, Arauca, Guayabero y Losada, y el río Tocantins (Figura 1).

Fase de campo. Las excursiones para la estimación poblacional se hicieron en embarcaciones de 14 a 43 metros de eslora con plataformas de observación que oscilaban entre los 2 y los 6 metros de altura. La variación se basó en el tamaño del río muestreado y su navegabilidad. Para estandarizar los muestreos, estos se realizaron durante los períodos climáticos de aguas en ascenso o descenso, lo que permitía cubrir diferentes tipos de hábitats. Se descartó el monitoreo en aguas bajas debido a que las playas y bajos no permitían la navegación de la embarcación ni el acceso a tributarios y lagunas. Asimismo, se descartó el período de mayor inundación, ya que cientos de kilómetros de selva se inundan, y los delfines ingresan allí haciendo muy difícil su detección en el canal principal.

Los hábitats se estratificaron para generar un reporte de densidad diferencial y con un sustento ecológico. Los hábitats considerados fueron: río principal, tributarios, lagunas, islas, canales y confluencias, según la definición y tipificación de Trujillo, et al. (2011). El muestreo se diseñó combinando transectos en banda (paralelos a las orillas) y lineales (atravesando diagonalmente el río) según la metodología desarrollada por Gómez, et al. (2011; 2012) y Williams, et al. (2015). La velocidad de desplazamiento estuvo en el rango de 8 a 12 $\mathrm{km} /$ hora y el muestreo se hizo entre las 5:30 y las 17:30 horas. 


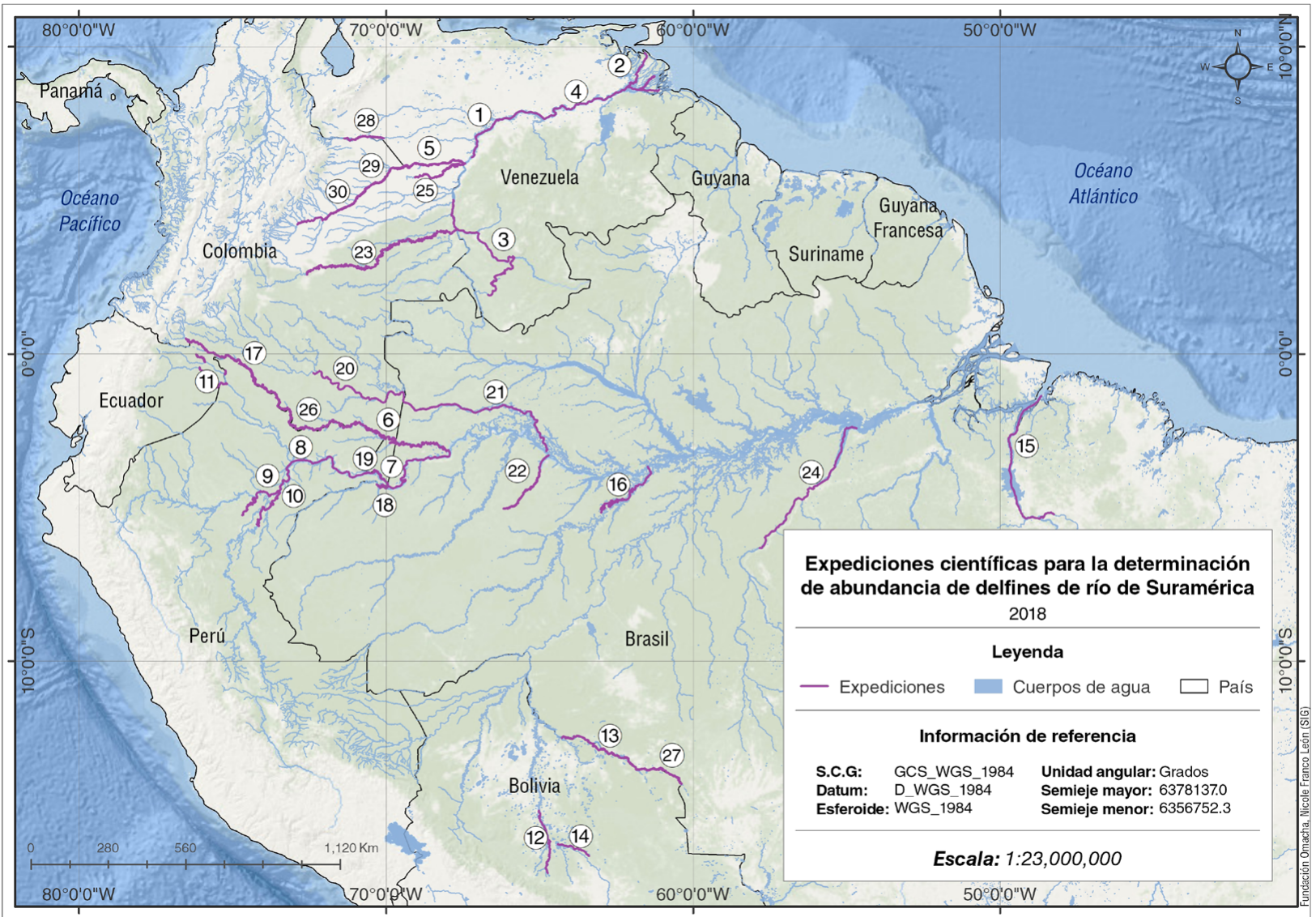

Figura 1. Expediciones científicas para la determinación de la abundancia de delfines de río en Suramérica. Los ríos se señalizaron con la siguiente numeración: 1. Orinoco (Medio), 2. Orinoco (Delta), 3. Orinoco (Casiquiare), 4. Orinoco (Venezuela), 5. Meta (Colombia), 6. Putumayo (Colombia), 7. Amazonas (Colombia), 8. Napo (Perú), 9. Marañón (Perú), 10. Ucayali (Perú), 11. Napo, Cuyabeno, Yasuní y Lagartococha (Ecuador), 12. Mamoré (Bolivia), 13. Iténez-Guaporé (Bolivia, Brasil), 14. Grande (Bolivia), 15. Tocantins (Brasil), 16. Madeira (Brasil), 17. Putumayo (Colombia), 18. Javari (Brasil), 19. Loretoyacu (Colombia), 20. Caquetá-Japurá (Colombia, Brasil), 21. Caquetá-Japurá (Colombia, Brasil), 22. Purús (Brasil), 23. Guaviare (Colombia), 24. Tapajos (Brasil), 25. Bita (Colombia), 26. Putumayo (Colombia), 27. Iténez-Guaporé (Bolivia, Brasil), 28. Arauca (Colombia), 29. Meta (Colombia), y 30. Guayabero y Losada (Colombia).

La longitud de las transectos en banda se estandarizó en $2,5 \mathrm{~km}$ en tanto que los lineales oscilaron entre $1 \mathrm{y}$ $2,5 \mathrm{~km}$ según el ancho del río. Los transectos tuvieron un ancho de banda (w) de 200 metros. En las embarcaciones se instalaron dos plataformas de observación, una en proa y otra en popa, con seis investigadores a cargo de la detección de los delfines y de su registro. Todos los observadores recibieron capacitación previamente y asistencia durante las expediciones. Las posiciones de los investigadores se rotaban cada dos horas en el sentido de las manecillas del reloj. Cada una de las plataformas se consideró independiente, y solo el anotador de esfuerzo conocía las diferentes observaciones de cada plataforma y las registraba con códigos de asignación.

En cada observación se tomó el ángulo de la observación usando un compás, y se estimó la distancia al delfín. Igualmente, para cada observación se anotó la especie (Inia o Sotalia), la distancia a la orilla (en rangos de 0-50 m, 50$100 \mathrm{~m}, 100-150 \mathrm{~m}$ y $150-200 \mathrm{~m}$ ), y el tipo de orilla (tierra firme, selva inundada, arbustos, playa, barranco, rocas, asentamientos humanos, pasto flotante). Además, se registraron las coordenadas geográficas de cada observación usando un GPS Garmin ${ }^{\circledR}$ de referencia 64sc, así como las condiciones ambientales como el brillo solar, el estado del río, el tipo de río (aguas negras, blancas o mixtas, según criterios limnológicos).

Estimaciones de densidad y tamaño poblacional. Se calculó la densidad de cada especie, inicialmente en los transectos lineales, usando el programa Distance, versiones 5.0 y 6.0 (Bukland, et al., 2001, Thomas, et al., 2010) y la siguiente fórmula:

$$
\mathrm{D}=\frac{n E(i) f(0),}{2 \operatorname{Lg}(0)}
$$

donde $n$ es el número de grupos de delfines observados, $E(i)$ es la media grupal observada en el hábitat tipo $(i), f(0)$ es la probabilidad de detección de densidad a una distancia perpendicular $0, L$ es la longitud del transecto, y $g(0)$, la probabilidad de detección de un grupo en la línea de transecto. 
Para $\mathrm{g}(0)$ se siguieron las asunciones propuestas por Gómez, et al., (2011), al igual que el cálculo de la varianza. Para la función de detección, se analizaron tres modelos diferentes con el programa Distance: el uniforme, el medio normal (half-normal) y el de índice de riesgo, seleccionados siguiendo el criterio de información de Akaike (Akaike's information criterion, AIC).

Teniendo en cuenta que se encontró un patrón de gradiente de densidad con respecto a la orilla, con más delfines cerca de esta y menos hacia el centro del río, se calculó la probabilidad de detección $g(0)$ de manera escalada usando los rangos de distancia $(0-50 \mathrm{~m}, 50-100 \mathrm{~m}, 100-150 \mathrm{~m} \mathrm{y}$ 150-200 m).

El tamaño de la población de delfines de río (Ni) para cada hábitat se calculó con la ecuación $\mathrm{Ni}=\mathrm{AiDi}$.

Para construir mapas que mostraran la heterogeneidad de la abundancia de los delfines en las subcuencas hidrográficas, se construyeron rangos de amplitud (alto, medio y bajo) para los tamaños poblacionales calculados con las siguientes ecuaciones:

$$
\mathrm{K}=1+3.2222(\log 10(\mathrm{n})),
$$

donde $\mathrm{K}$ es el número de intervalos de clase $(\mathrm{k})$, y

$$
\mathrm{W}=(\text { Valor máximo-Valor mínimo }) / \mathrm{K} \text {, }
$$

donde $\mathrm{W}$ es el rango de amplitud.

Cada rango se calculó para todas las dos especies y por subcuenca.

Especialización cartográfica de la información. Las salidas gráficas se construyeron empleando el programa ArcGIS 10.2.2 (Environmental Systems Research Institute, ESRI, 2014), se usaron conjuntos de datos georreferenciados (vector y ráster) a varias escalas, incluidas las redes fluviales, los límites de la cuenca, las direcciones de drenaje y las acumulaciones de flujo proporcionados por la plataforma HydroSHEDS (U.S. Geological Service, USGS, 2018). La base de datos de hidroeléctricas en Brasil, Bolivia, Colombia, Ecuador, Perú y Venezuela se construyó siguiendo la propuesta de Latrubesse, et al., (2017) y Anderson, et al., (2018).

\section{Resultados}

Estimaciones de densidad y tamaño poblacional. En total se han llevado a cabo 30 expediciones científicas en seis países cubriendo las cuencas del Amazonas, el Orinoco y el Tocantins. En la cuenca Amazónica se muestrearon 19 ríos, en la del Orinoco, seis y en el Tocantins, uno. En total se cubrieron $28.760 \mathrm{~km}$.

En general, las densidades más bajas se reportaron para los ríos ecuatorianos de la cuenca del Amazonas (Inia: 0,2 ind $/ \mathrm{km}$ y Sotalia: 0,07 ind $/ \mathrm{km}$ ). En segundo lugar, sobresalió el río Bita (Inia: 0,06 ind $/ \mathrm{km}$ ). Una situación similar ocurre en el río Tocantins, donde se reporta solo la presencia de $I$. araguiaensis, con valores bajos $(0,93 \mathrm{ind} / \mathrm{km})$. Por otro lado, los ríos con mayores densidades corresponden al
Purús en Brasil (6,7 ind $/ \mathrm{km})$, Samiria en Perú (5,94 ind $/ \mathrm{km})$ e Iténez entre Bolivia y Brasil $(8,1 \mathrm{ind} / \mathrm{km})$. Uno de los países mejor muestreado hasta la fecha es Colombia, donde se han evaluado los ríos Arauca, Meta, Guayabero, Losada, Bita, Guaviare, Caquetá, Putumayo y Amazonas (Tablas 1 y 2).

Los tamaños poblacionales más bajos para el género Inia se han reportado en los ríos ecuatorianos Napo, Cocayá y Tiputini de la cuenca del Amazonas. En segundo lugar se encuentra el río Bita, en la cuenca del Orinoco colombiano. Una situación similar ocurre en el río Tocantins, donde se reporta solo la presencia de la especie recientemente descrita I. araguiaensis. Por otro lado, los ríos con mayores tamaños poblacionales para $I$. g. geoffrensis fueron Purús y Tapajos (Brasil), Amazonas, Loretoyacú, y Javarí (Colombia), y Samiria y Marañón (Perú), y para I. boliviensis los ríos Iténez (Bolivia/Brasil) y Mamoré (Bolivia). En el caso del género Sotalia, los ríos con mayores tamaños poblacionales de la especie fluvial (S. fluviatilis) fueron el Purús y el Tapajos (Brasil), el Amazonas, el Loretoyacú y el Javarí (Colombia), y el Samiria y el Marañón (Perú), y para el morfotipo costero (Sotalia sp.) fue el delta del río Orinoco (Tabla 3).

Especialización cartográfica de la información. Los tamaños poblaciones calculados para las especies y subespecies del género Inia se categorizaron por rangos para cada una de las cuencas evaluadas y espacializadas en salidas gráficas. Se obtuvieron valores bajos para los ríos Guaviare y Orinoco (Casiquiare) en la cuenca del río Orinoco; Putumayo, Caquetá, Napo, Grande e Iténez en la cuenca del Amazonas, y el río Tocantins. Se registraron valores medios en el delta del río Orinoco, en los ríos Meta y Arauca en la cuenca del Orinoco, y en los ríos Marañón, Ucayali y Mamoré en la cuenca del Amazonas. Por último, se reportaron valores altos en la cuenca media del Orinoco, y en los ríos Purús, Tapajos e Iténez en la cuenca del Amazonas (Figura 2).

Se obtuvieron valores bajos para Sotalia fluviatilis en los ríos Putumayo/Iça y Napo en la cuenca del Amazonas y en la cuenca baja del río Tocantins. Se registraron valores medios para Sotalia sp. en la cuenca media y en el delta del río Orinoco y para $S$. fluviatilis en los ríos Caquetá/ Japurá y Curaray en la cuenca del Amazonas. Por último, se reportaron valores altos en los ríos Marañón, Ucayali y Purús en la cuenca del Amazonas (Figura 3).

\section{Discusión}

Estimaciones de densidad y tamaño poblacional. La iniciativa suramericana para la estimación poblacional de delfines de río es, hasta el momento, la más completa y la de mayor cubrimiento geográfico para delfines de río a nivel global. Los resultados coinciden con lo expuesto por Gómez, et al., (2011; 2012; 2012a); Mosquera-Guerra, et al., (2015) y Pavanato, et al., (2016), en cuanto a: i) el hallazgo de valores heterogéneos en la densidad de individuos por tipo de hábitat; ii) los tamaños poblacionales por cuenca, y iii) los rangos de distribución longitudinales, latitudinales 
Tabla 1. Densidades estimadas $(D)$ para las especies del género Inia en los ecosistemas acuáticos evaluados para cada tipo de hábitat utilizando transectos de ancho de banda de $200 \mathrm{~m}$. No se obtuvo información en campo (espacios en blanco) para algunas categorías.

\begin{tabular}{|c|c|c|c|c|c|c|c|c|}
\hline \multirow[t]{3}{*}{ Cuenca } & \multirow[t]{3}{*}{ Ríos } & \multirow[t]{3}{*}{ Año } & \multicolumn{5}{|c|}{ Tipos de hábitats } & \multirow[b]{2}{*}{ Confluencias } \\
\hline & & & Río principal & Tributario & Canal & Islas & Lagunas & \\
\hline & & & $D$ (ind/km) & $D$ (ind/km) & $D$ (ind/km) & $D$ (ind/km) & $D($ ind $/ \mathbf{k m})$ & $D$ (ind/km) \\
\hline \multirow{20}{*}{ Amazonas } & Inia boliviensis & & & & & & & \\
\hline & Bolivia (Iténez) & 2007 & & 3,21 & 2,94 & & 8,1 & \\
\hline & Bolivia (Mamoré) & 2007 & 3,52 & & & & & \\
\hline & Bolivia (Grande) & 2011 & & 2,4 & 5,6 & & 8,1 & 6,5 \\
\hline & Bolivia (Iténez) & 2017 & 0,7 & & & & & 0,6 \\
\hline & Inia g. geoffrensis & & & & & & & \\
\hline & $\begin{array}{l}\text { Colombia (Amazonas, } \\
\text { Loretoyacú y Javarí) }\end{array}$ & 2007 & 1,82 & 3,77 & 2,58 & 1,91 & 18,4 & 12,8 \\
\hline & Colombia (Putumayo) & 2017 & 0,3 & 1 & 0,7 & 0,6 & & 0,8 \\
\hline & $\begin{array}{l}\text { Ecuador (Napo, } \\
\text { Cuyabeno, Yasuní y } \\
\text { Lagartococha) }\end{array}$ & 2006 & & 2,78 & & & 0,56 & 2,87 \\
\hline & Ecuador (Napo) & 2017 & 1,85 & & & & & \\
\hline & Ecuador (Cocayá) & 2017 & & 1,41 & & & & \\
\hline & Ecuador (Tiputini) & 2017 & & 1,06 & & & & \\
\hline & Ecuador (Yasuní) & 2017 & & 1,51 & & & & \\
\hline & $\begin{array}{l}\text { Perú } \\
\text { (Samiria y Marañón ) }\end{array}$ & 2006 & 2,72 & 5,94 & 4,92 & & & 4,22 \\
\hline & $\begin{array}{l}\text { Perú (Confluencia } \\
\text { Napo-Santa Rosa) }\end{array}$ & 2015 & 1,18 & 1,96 & 1,21 & 2,25 & 3,47 & 10,83 \\
\hline & Brasil (Purús) & 2012 & 3,7 & 6,7 & 4,8 & 1,6 & 3,02 & 3,5 \\
\hline & $\begin{array}{l}\text { Brasil-Colombia } \\
\text { (Japurá-Caquetá, } \\
\text { Solimoes) }\end{array}$ & 2014 & 1,26 & 1,96 & 1,47 & 1 & 3,31 & 2,2 \\
\hline & Brasil (Tapajos) & 2014 & 0,87 & 2,59 & 1,85 & 5,7 & & \\
\hline & Inia araguaiaensis & & & & & & & \\
\hline & Brasil (Tocantins) & 2014 & 0,93 & & 1,86 & 0,7 & & \\
\hline \multirow{9}{*}{ Orinoco } & Inia g humboldtiana & & & & & & & \\
\hline & Colombia (Meta) & 2008 & 0,57 & & 1,96 & 0,6 & & 9,29 \\
\hline & Colombia (Guaviare) & 2016 & 0,7 & 0,45 & & & & 6,3 \\
\hline & $\begin{array}{l}\text { Colombia } \\
\text { (Guayabero/Losada) }\end{array}$ & 2016 & 0,89 & & 0,38 & & & 0,58 \\
\hline & Colombia (Arauca) & 2017 & 2,6 & & & & & 8,3 \\
\hline & Colombia (Bita) & 2016 & & 0,56 & & & & 7 \\
\hline & $\begin{array}{l}\text { Venezuela } \\
\text { (Orinoco/ Medio) }\end{array}$ & 2013 & 1,14 & & 0,28 & 1,3 & & 9,96 \\
\hline & $\begin{array}{l}\text { Venezuela } \\
\text { (Orinoco/Delta) }\end{array}$ & 2006 & 0,6 & 1,2 & 0,8 & & & 0,6 \\
\hline & $\begin{array}{l}\text { Venezuela } \\
\text { (Orinoco/Casiquiare) }\end{array}$ & 2013 & 0,03 & & & & & \\
\hline
\end{tabular}


(planos de inundación) y altitudinales para estos cetáceos en los ecosistemas acuáticos evaluados en la Amazonia y la Orinoquia, y para cada una de las especies existentes.

Se comprobó la importancia de los niveles de productividad de los sistemas para los valores de densidad y tamaño poblacional que aquí se reportan. Un ejemplo de esta condición son los valores de densidades y tamaños poblacionales más bajos para Inia y Sotalia reportados en los ríos Cuyabeno, Aguarico, Yasuní, Cocaya y Tiputini en Ecuador, los cuales corresponden limnológicamente a ríos de aguas negras, y donde no existen pesquerías de carácter comercial debido a la baja biomasa de peces. En contraste, en los ríos de aguas blancas como el Iténez (Bolivia/Brasil) y Purús (Brasil), los valores de densidad fueron altos, lo que indica la calidad del hábitat y la disponibilidad de presas necesarios para albergar el alto número de delfines registrado.

En las tres cuencas se resalta la presencia de áreas protegidas y la baja actividad humana, especialmente la pesca (Saunders, et al., 2002; Gómez, et al., 2011), aspecto que explicaría parcialmente los mayores valores de densidad de individuos reportados en algunas cuencas como la del Purús, Samiria e Iténez. Las densidades más altas registradas fueron las de $I$. g. geoffrensis (hasta 18 delfines $/ \mathrm{km}^{2}$ en canales de los planos de inundación) en la Reserva de Desarrollo Sustentable Mamiragua en Brasil (Martin \& da Silva, 2004), que es una de las áreas protegidas de mayor extensión en la Amazonia (Pires, 2006). Ello sugiere que las mayores densidades y tamaños poblacionales de delfines de río parecen encontrarse en las áreas protegidas con ecosistemas acuáticos bien administrados (Gómez, et al., 2011).

A escala regional, se sugiere que los hábitats esenciales para los delfines de río se encuentran dentro de los $200 \mathrm{~m}$ de las riberas, como ocurre con las confluencias y hábitats lacustres (complejo de humedales), donde se concentran los peces (Vidal, et al., 1997, Trujillo, 2000; Trujillo, et al., 2016; Martin \& da Silva, 2004; Gómez, et al., 2011; 2012; Mosquera-Guerra, et al., 2015), lugares estos compartidos con los pescadores donde, generalmente, los delfines de río se ven atrapados en redes. Situaciones similares han sido reportadas por Smith, et al., (2006) en el río Ganges, donde los delfines de río (Platanista gangetica) ocupan preferentemente estas áreas de ribera.

Corroboramos los resultados de investigaciones previas de nuestro grupo y de otros investigadores en las cuales se señala que los valores de densidad de los depredadores tope disminuyen con el aumento de los factores de estrés

Tabla 2. Densidades estimadas $(D)$ para las especies del género Sotalia en los ecosistemas acuáticos evaluados para cada tipo de hábitat (río principal, tributario, canal, islas, lagunas, confluencias), utilizando transectos de ancho de banda de $200 \mathrm{~m}$. No se obtuvo información en campo (espacios en blanco) para algunas categorías.

\begin{tabular}{|c|c|c|c|c|c|c|c|c|}
\hline \multirow[t]{3}{*}{ Cuenca } & \multirow[t]{3}{*}{ Ríos } & \multirow[t]{3}{*}{ Año } & \multicolumn{6}{|c|}{ Tipos de hábitats } \\
\hline & & & Río principal & Tributario & Canal & Islas & Lagunas & Confluencias \\
\hline & & & $D$ (ind/km) & D (ind/km) & $D$ (ind $/ \mathbf{k m})$ & $D$ (ind $/ \mathbf{k m})$ & $D$ (ind/km) & $D$ (ind/km) \\
\hline \multirow{9}{*}{ Amazonas } & Sotalia fluviatilis & & & & & & & \\
\hline & $\begin{array}{l}\text { Colombia } \\
\text { (Amazonas, } \\
\text { Loretoyacú y Javarí) }\end{array}$ & 2007 & 3,35 & 4,21 & 5,1 & 1,74 & 12,32 & 28,14 \\
\hline & $\begin{array}{l}\text { Colombia } \\
\text { (Putumayo) }\end{array}$ & 2017 & 0,1 & 0,9 & 0,6 & 0,5 & & 0,6 \\
\hline & $\begin{array}{l}\text { Ecuador (Napo, } \\
\text { Cuyabeno, Yasuní y } \\
\text { Lagartococha) }\end{array}$ & 2006 & & 0,28 & & & & 1,97 \\
\hline & $\begin{array}{l}\text { Perú (Samiria y } \\
\text { Marañón ) }\end{array}$ & 2006 & 4,87 & 6,08 & 3,09 & 2,1 & & 8,69 \\
\hline & $\begin{array}{l}\text { Perú (Confluencia } \\
\text { Napo-Santa Rosa) }\end{array}$ & 2015 & 2,1 & 1,2 & 1,28 & & & 3,8 \\
\hline & Brasil (Purús) & 2012 & & & & & & \\
\hline & $\begin{array}{l}\text { Brasil-Colombia } \\
\text { (Japurá-Caquetá, } \\
\text { Solimoes) }\end{array}$ & 2014 & & & & & & \\
\hline & Brasil (Tapajos) & 2014 & 0,85 & & 1,57 & 1,63 & & \\
\hline \multirow[b]{2}{*}{ Orinoco } & Sotalia sp. & & & & & & & \\
\hline & $\begin{array}{l}\text { Venezuela } \\
\text { (Orinoco/Medio) }\end{array}$ & 2006 & 1,06 & & & & & 1,41 \\
\hline
\end{tabular}


de origen humano, aunque con ciertas evidencias de resiliencia y plasticidad en el caso de los delfines (Sandin, et al., 2008; Baum \& Worm, 2009; Gómez, et al., 2012; Mosquera-Guerra, et al., 2015a). Para el caso de los delfines de río suramericanos, Trujillo, et al., (2010a) han reportado tensores de origen antrópico que influencian los parámetros poblacionales, tales como la degradación de los hábitats por pérdida de la conectividad, la contaminación, las interacciones negativas con pesquerías, el incremento en el tráfico de embarcaciones, el cambio climático y la construcción de infraestructura hidroeléctrica.

Trujillo, et al., (2011); Gómez, et al., 2012a y Mosquera-Guerra, et al., (2015a) reportaron, además, que las variaciones en los valores de la densidad, el tamaño de grupo y la población de los delfines de río, se relacionaban con factores ecológicos en las cuencas como los pulsos de

Tabla 3. Tamaños poblacionales para las especies de los géneros Inia y Sotalia en los ecosistemas acuáticos estudiados asociados con las cuencas de los ríos Amazonas y Orinoco. Abreviaturas: (a) Gómez, et al., 2012; (b) Pavanato, et al., en prensa; (c) Mosquera-Guerra, et al., 2015d; (d) Mosquera-Guerra, et al., 2015a y (e) Pavanato, et al., 2016.

\begin{tabular}{|c|c|c|c|c|c|c|c|c|}
\hline \multirow[t]{2}{*}{ Cuenca } & \multirow[t]{2}{*}{ País } & \multirow[t]{2}{*}{ Año } & \multicolumn{6}{|c|}{ Tamaño de la población } \\
\hline & & & I. g. geoffrensis & I. g. humboldtiana & I. boliviensis & I. araguaiaensis & S. fluviatilis & Sotalia sp. \\
\hline \multirow{16}{*}{ Amazonas } & Bolivia (Iténez) & 2007 & & & $3201(0,40)$ a & & & \\
\hline & Bolivia (Mamoré) & 2007 & & & $1369(1,14) \mathrm{a}$ & & & \\
\hline & Bolivia (Grande) & 2011 & & & $523(0,05)$ & & & \\
\hline & Bolivia (Iténez) & 2017 & & & $291(0,5)$ & & & \\
\hline & $\begin{array}{l}\text { Colombia (Amazonas, } \\
\text { Loretoyacú y Javarí) }\end{array}$ & 2007 & $1,115(0,78) \mathrm{a}$ & & & & $1,545(0,61) a$ & \\
\hline & Colombia (Putumayo) & 2017 & $546(0,22)$ & & & & $241(0,1)$ & \\
\hline & $\begin{array}{l}\text { Ecuador (Napo, } \\
\text { Cuyabeno, Yasuní y } \\
\text { Lagartococha) }\end{array}$ & 2006 & $147(1,38) \mathrm{a}$ & & & & $19(1,37) \mathrm{a}$ & \\
\hline & Ecuador (Napo) & 2017 & $14(1,1)$ & & & & & \\
\hline & Ecuador (Cocayá) & 2017 & $8(1,3)$ & & & & $12(1,6)$ & \\
\hline & Ecuador (Tiputini) & 2017 & $6(1,2)$ & & & & & \\
\hline & Ecuador (Yasuní) & 2017 & $9(1,4)$ & & & & & \\
\hline & $\begin{array}{l}\text { Perú (Samiria y } \\
\text { Marañón) }\end{array}$ & 2006 & $917(0,34) \mathrm{a}$ & & & & $1.319(0,43) \mathrm{a}$ & \\
\hline & $\begin{array}{l}\text { Perú (Confluencia Napo- } \\
\text { Santa Rosa) }\end{array}$ & 2015 & $429(0,93)$ & & & & $726(1,79)$ & \\
\hline & Brasil (Purús) & 2012 & $2.241(2,7)$ & & & & $1.672(2,23)$ & \\
\hline & $\begin{array}{l}\text { Brasil-Colombia (Japurá- } \\
\text { Caquetá, Solimoes) }\end{array}$ & 2014 & 503 b y c & & & & $666 \mathrm{~b}$ & \\
\hline & Brasil (Tapajos) & 2015 & $1.815(0,4) \mathrm{e}$ & & & & & \\
\hline Tocantins & Brasil (Tocantins) & 2014 & & & & $363(1,45)$ & $240(3,58)$ & \\
\hline \multirow{8}{*}{ Orinoco } & Colombia (Meta) & 2008 & & $1.016(0,85) \mathrm{a}$ & & & & \\
\hline & Colombia (Guaviare) & 2016 & & $370(1,6) d$ & & & & \\
\hline & $\begin{array}{l}\text { Colombia } \\
\text { (Guayabero/Losada) }\end{array}$ & 2016 & & $15(1,9) \mathrm{d}$ & & & & \\
\hline & Colombia (Arauca) & 2017 & & $400(0,1)$ & & & & \\
\hline & Colombia (Bita) & & & $27(0,3)$ & & & & \\
\hline & $\begin{array}{l}\text { Venezuela } \\
\text { (Orinoco/Casiquiare) }\end{array}$ & 2013 & & 188 & & & & \\
\hline & $\begin{array}{l}\text { Venezuela } \\
\text { (Orinoco/ Medio) }\end{array}$ & 2013 & & $1.779(0,87) \mathrm{a}$ & & & & \\
\hline & $\begin{array}{l}\text { Venezuela } \\
\text { (Orinoco/Delta) }\end{array}$ & 2006 & & $894(1,89)$ & & & & $881(1,56)$ \\
\hline Total & & & 7.750 & 4.689 & 5.384 & 363 & 6.440 & 881 \\
\hline
\end{tabular}




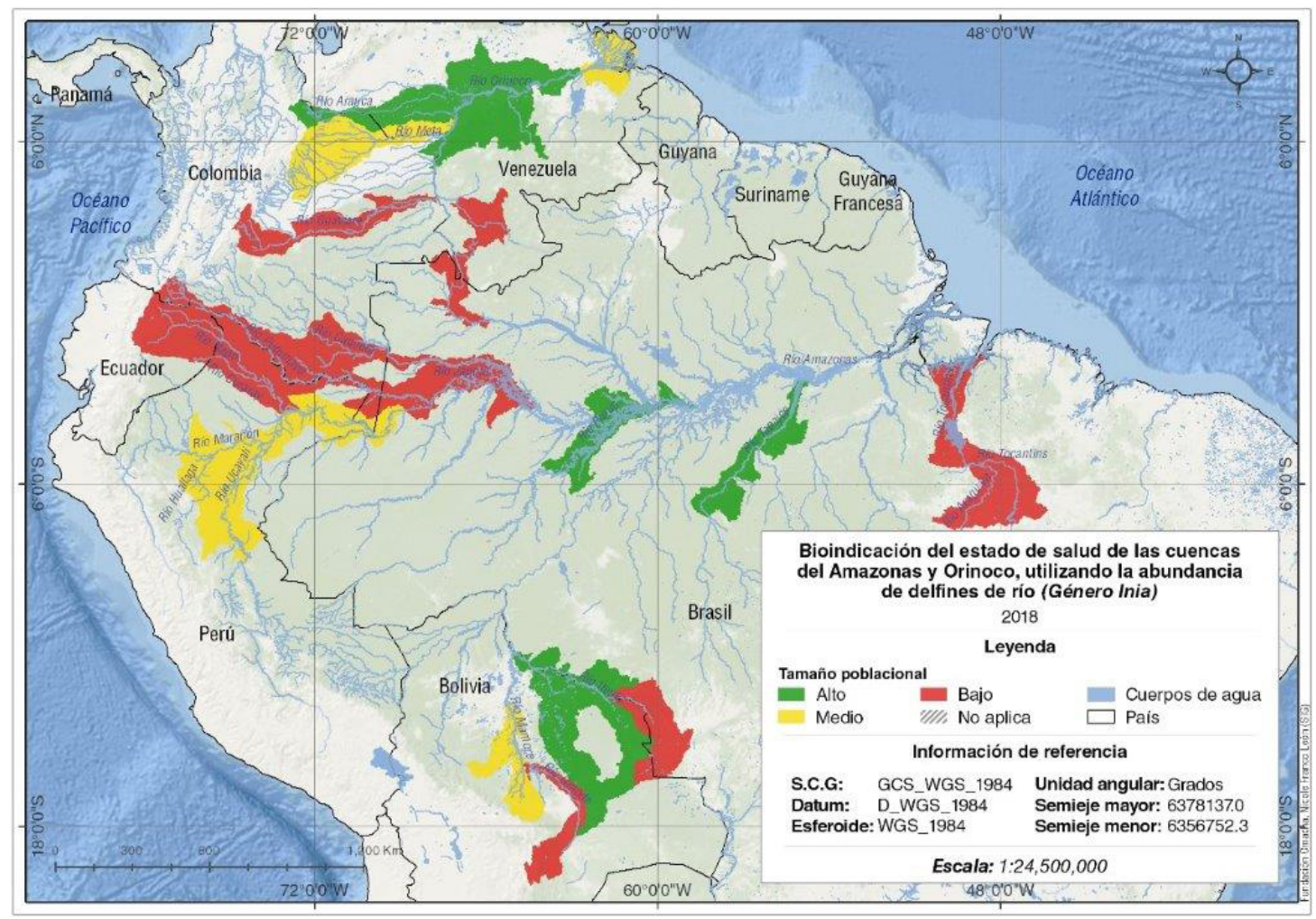

Figura 2. Bioindicación del estado de salud de las cuencas del Amazonas y el Orinoco utilizando los tamaños poblacionales de delfines de río del género Inia

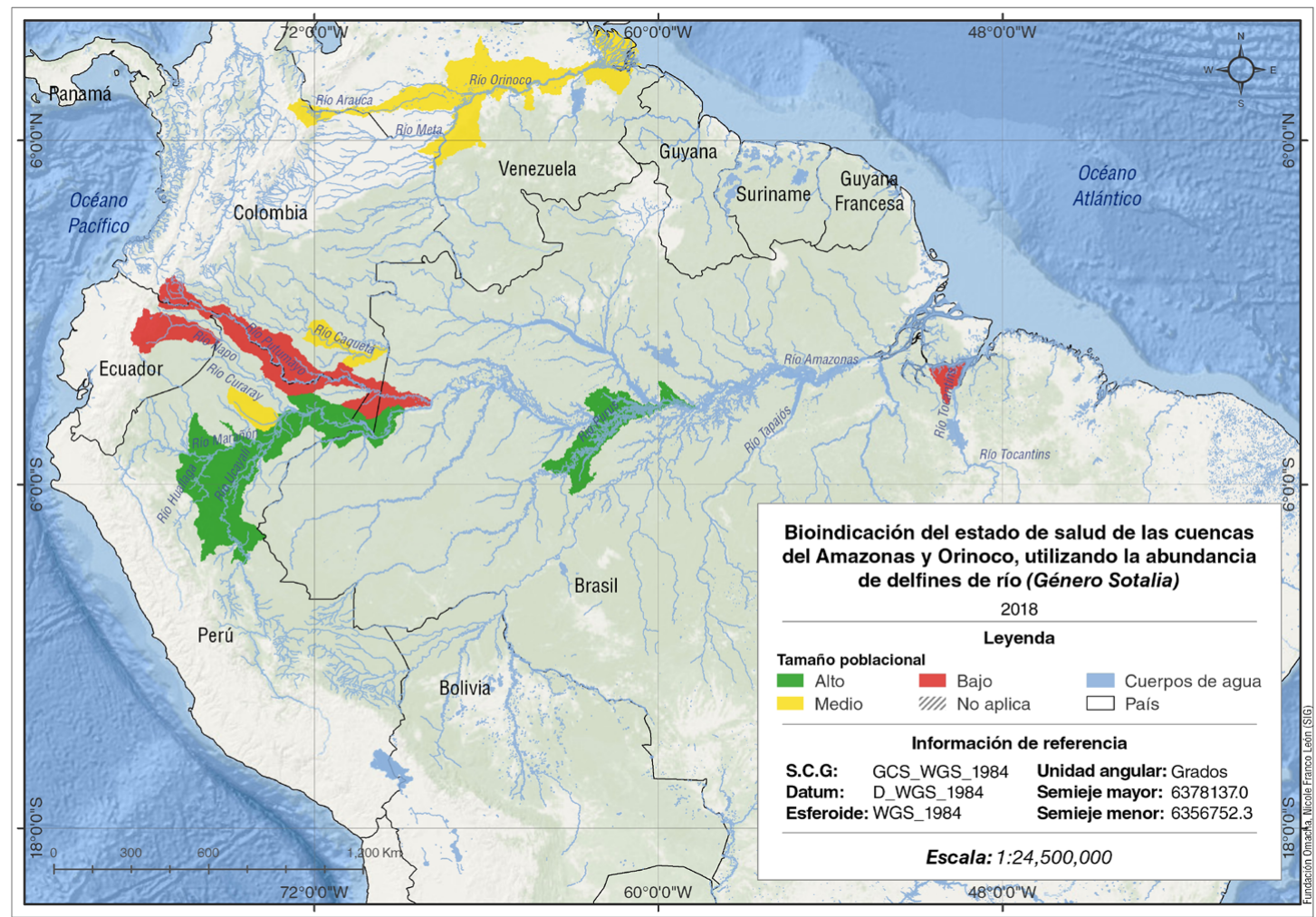

Figura 3. Bioindicación del estado de salud de las cuencas del Amazonas y Orinoco utilizando los tamaños poblacionales de delfines de río del género Sotalia 
inundación, lo que obliga a los delfines en aguas bajas a concentrarse en los canales principales y a dispersarse en aguas altas en aquellos ambientes acuáticos asociados al bosque inundando a donde se desplazan detrás de los peces.

Espacialización cartográfica de la información. Gómez, et al., (2012a) discuten sobre las múltiples ventajas que presenta el uso de las especies de delfines de río como indicadoras del grado de degradación de los ecosistemas acuáticos donde habitan, e incluyeron los siguientes elementos de importancia en su condición bioindicadora: (1) los delfines de río se distribuyen en todos los tipos de hábitats de las cuencas de los ríos Amazonas y Orinoco, con excepción de los rápidos y las áreas con ecosistemas muy degradados (por ejemplo, el río Napo); (2) los delfines de río son relativamente fáciles de observar en un ecosistema donde la mayoría de las especies se encuentran bajo aguas de gran turbidez; (3) las evaluaciones de los delfines de río son relativamente fáciles de realizar y se requiere un menor procesamiento de las muestras que en el caso de otros indicadores potenciales como los macroinvertebrados acuáticos y las algas; (4) los delfines de río tienen una larga vida y, por lo tanto, permiten evaluar los riesgos a largo plazo de la degradación de los ecosistemas acuáticos; (5) los delfines de río pueden actuar como especies centinela al proporcionar advertencias tempranas sobre los aumentos actuales o futuros en la degradación del ecosistema debidos a la bioacumulación de metales pesados como el mercurio $(\mathrm{Hg})$, según lo reportado en las investigaciones de Rosas \& Kesä, (1996); MosqueraGuerra, et al., (2015b) y Mosquera-Guerra, et al., (2018a), y, por último, (6) los delfines de río son mamíferos carismáticos, por lo tanto, pueden emplearse como especies emblemáticas para aumentar la conciencia pública sobre temas de conservación y manejo de los ecosistemas acuáticos (Sergio, et al., 2008). Todas estas ventajas, hacen que un análisis espacial a escala regional de las cuencas permita evidenciar áreas en buenas condiciones de conservación y otras con amenazas concretas que merecen ser evaluadas y abordadas, así como asociar bases de datos a sistemas de información geográfica que puedan ser actualizadas de manera permanente.

La bioindicación de las cuencas hidrográficas empleando los tamaños poblacionales para especies de los géneros Inia y Sotalia (Figuras 4 y 5), evidencia los fuertes procesos de degradación en el río Guaviare y en el brazo Casiquiare en la cuenca del Orinoco, en los ríos Caquetá/Japurá, Putumayo/ Iça, Napo, Grande, en la cuenca alta del Iténez y en la cuenca del río Tocantins. Estos resultados coinciden con lo expuesto por Trujillo, et al., (2010) y Mosquera-Guerra, et al., (2018) en cuanto a la presencia de intensos procesos de deforestación, de minería aurífera y de pesca excesiva, y en la cuenca alta de los ríos Iténez y Tocantins, además, el establecimiento de hidroeléctricas (Latrubesse, et al.,

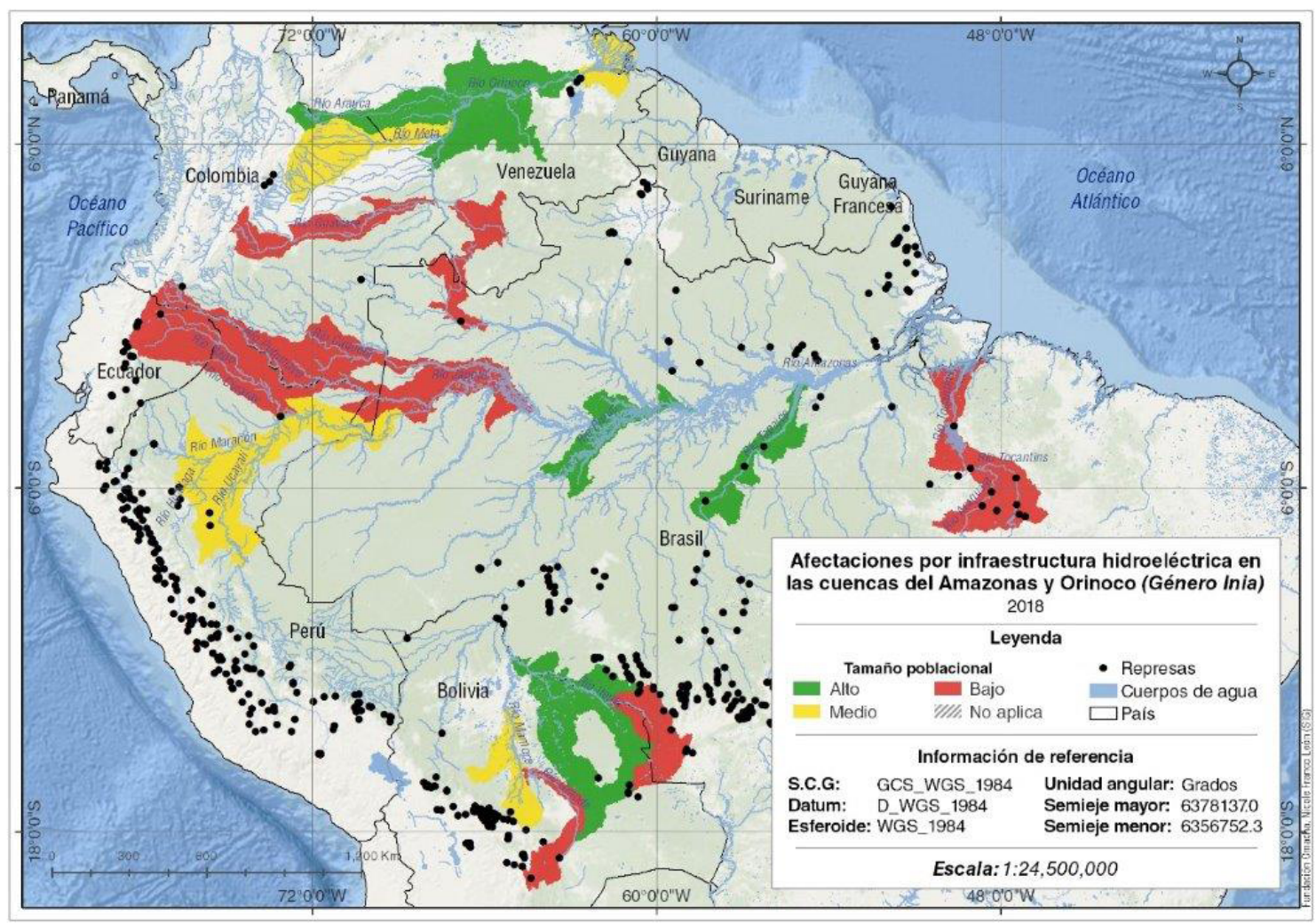

Figura 4. Infraestructura hidroeléctrica y bioindicación de los ecosistemas acuáticos empleando los tamaños poblacionales de las especies del género Inia en las cuencas de los ríos Amazonas y Orinoco 


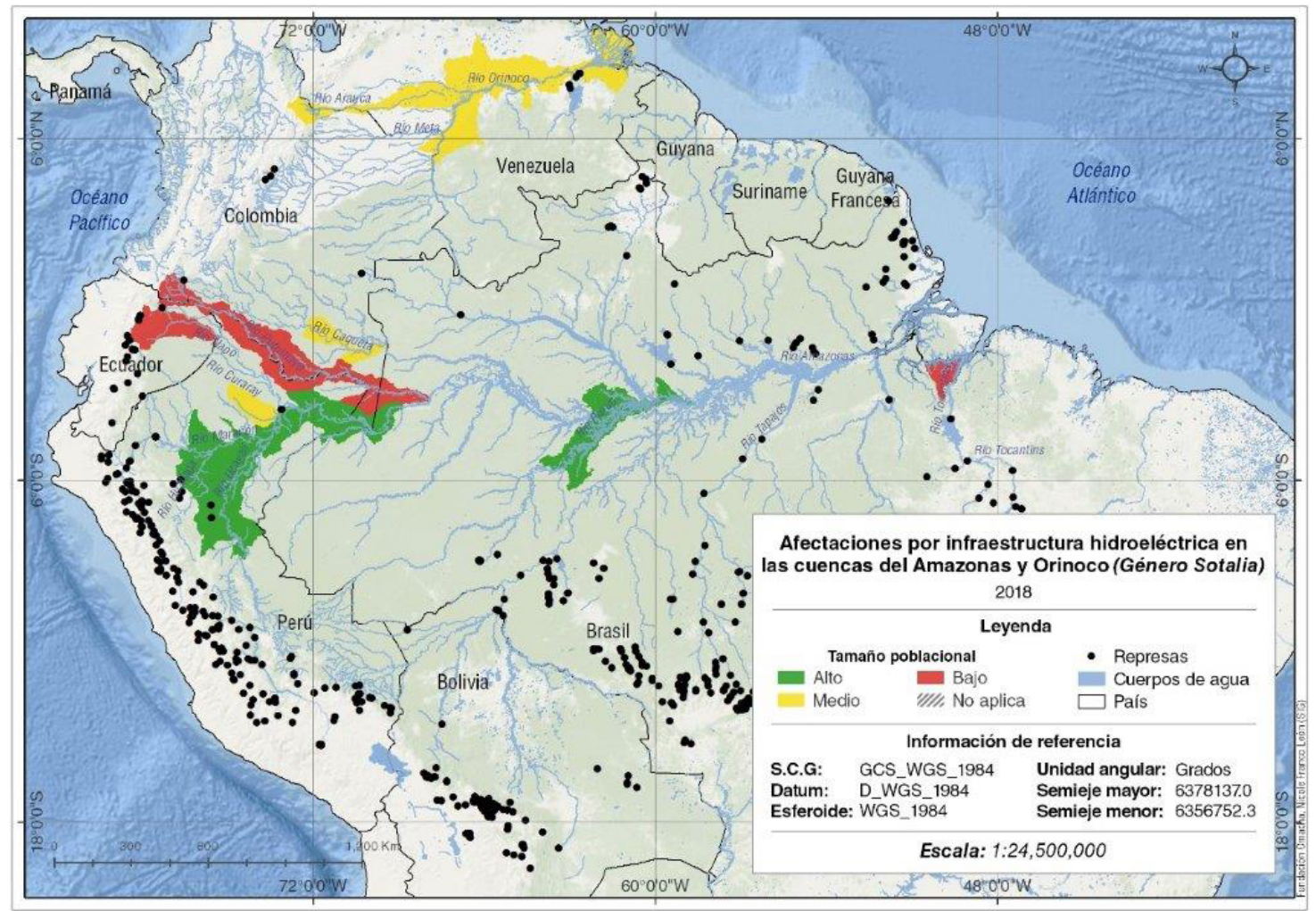

Figura 5. Infraestructura hidroeléctrica y bioindicación de los ecosistemas acuáticos empleando los tamaños poblacionales de las especies del género Sotalia en las cuencas de los ríos Amazonas y Orinoco

2017; Anderson, et al., 2018; Mosquera-Guerra, et al., 2018). Este tipo transformación de los ecosistemas acuáticos heterogéneos y complejos, especialmente en la Amazonia y la cuenca del Tocantins, como resultado de la construcción de más de un centenar de centrales hidroeléctricas provoca la homogeneización ecológica de estos sistemas, cambios en los pulsos de inundación aguas debajo de las represas, así como la retención de nutrientes como el nitrógeno y el fósforo, que limitan la productividad primaria en redes tróficas acuáticas (Forsberg, et al., 2017; Latrubesse, et al., 2017; Anderson, et al., 2018; Mosquera-Guerra, et al., 2018b). La presencia de este tipo de infraestructura también genera pérdidas significativas en la conectividad fluvial en cuencas como la del Amazonas, donde se presenta la mayor diversidad de delfines de río y peces del planeta (MosqueraGuerra et al., 2015; Anderson et al., 2018). Estos tensores han causado la transformación de extensas áreas ocupadas por los delfines de río en Suramérica. Arauho \& Wang, (2014) señalaron los efectos que tendría en la población de la especie recientemente descrita I. araguaiaensis (Hrbek, et al., 2014), la presencia de siete proyectos hidroeléctricos, entre estos la hidroeléctrica de Tucuruí, los cuales transformarían el 54,9\% de su área de distribución y restringirían los movimientos de la especie a tramos entre estos proyectos (Mosquera-Guerra, et al., 2018). Por otra parte, las especies I. boliviensis, I.g. geoffrensis y Sotalia fluviatilis se verían afectadas por tres nuevas hidroeléctricas en los ríos Marañón, Madeira y Ucayali.

La reducción del sedimento ha sido otro efecto negativo que se ha venido agudizando en las macrocuencas, según Forsberg, et al., (2017), quienes predicen que al entrar en operación las presas de Rositas, Angosto Del Bala, Inambari, Tam 40, Pongo De Aguierre y Pongo De Monseriche, se reducirá el suministro de sedimento en un $69 \%$, el de fósforo en un $67 \%$ y el de nitrógeno en $57 \%$, en tanto que en la región Andina y en toda la cuenca del Amazonas, las reducciones serán de 64,51 y $23 \%$, respectivamente. Dichas reducciones tendrán un impacto también en la geomorfología de los canales, la fertilidad de los planos de inundación y la productividad acuática (Latrubesse, et al., 2017). La eventual reducción de los niveles de biomasa en estos sistemas podría exacerbar los conflictos entre los delfines de río y las pesquerías artesanales y comerciales debido a la disminución de los recursos pesqueros en el Amazonas.

Por último, se reportan valores de bioindicación diferentes de los géneros Inia y Sotalia en los ecosistemas acuáticos del Amazonas colombiano, el peruano y el brasilero, resultados que probablemente se deban a las capturas dirigidas de la especie I.g.geoffrensis, utilizada como atrayente en las pesquerías de Calophysus macropterus (mota, simí, piracathinga) (Trujillo, et al., 2010; Mosquera-Guerra, et al., 2015c). 


\section{Conclusiones}

El proceso de estandarización del protocolo metodológico para la estimación poblacional de delfines ha tenido elementos positivos a lo largo de su construcción, como la realimentación entre investigadores, la cual ha resultado en sugerencias valiosas que lo han consolidado. En este sentido, es importante reconocer que en el futuro pueden hacerse otros ajustes, especialmente por la posibilidad de usar herramientas que mejoren los conteos, por ejemplo, la que ya se está implementando con drones, especialmente en zonas de confluencias y lagunas, donde la transparencia del agua permite contar de manera más precisa el número de delfines avistados y ajustar, así, el coeficiente de detección de los observadores.

El programa de conservación de delfines de agua dulce en Suramérica ha mostrado ser una herramienta contundente de manejo al incorporar la estimación poblacional, la capacitación y la creación de redes de trabajo, así como la construcción y la implementación de planes de manejo, y la articulación de la academia, las ONG y los gobiernos de la región.

Uno de los resultados concretos de la información recolectada durante los últimos 12 años fue la revisión de la categoría de amenaza de Inia geoffrensis por parte de la IUCN, que la modificó de "Datos deficientes" (DD) a "En peligro" (EN).

Por último, se corroboró la utilidad de los delfines de río como especies indicadoras y centinelas del estado de salud de los ecosistemas acuáticos en los cuales se distribuyen, y su uso potencial como valores objeto de conservación de las áreas protegidas de la Amazonia y la Orinoquia.

\section{Agradecimientos}

Esta iniciativa no habría sido posible sin el apoyo y liderazgo de la Fundación Omacha, del World Wildlife Fund, el Whitley Fund for Nature, Whale and Dolphin Conservation, National Geographic, el Instituto Alexander von Humbold, el Ministerio de Ambiente y Desarrollo Sostenible de Colombia, la Wildlife Conservation Society en Ecuador, Perú y Bolivia, Faunagua, Solinia, la Fundación La Salle de Venezuela, de Colciencias a través de la "Beca Doctorados Nacionales Convocatoria 785", y de las comunidades locales. Debemos especial agradecimiento a Victor Utreras, Mariana Escobar, Paul Van Damme, Cedric Gillman, Enrique Crespo y Marcela Portocarrero, así como a la dedicación de Catalina Gómez-Salazar, Mariana Farías, Heloise Pavanato, Miriam Marmontel, Saulo Usma y Marcelo Oliveira, entre otros.

\section{Contribución de los autores}

Fernando Trujillo-González: análisis de la información, organización de las bases de datos y generación de la cartografía; Federico Mosquera-Guerra: diseño metodológico, trabajo de campo y análisis de la información; Nicole Franco: trabajo de campo y análisis de la información.

\section{Conflicto de intereses}

No tenemos conflictos de intereses para reportar.

\section{Referencias}

Anderson, E. P., Jenkins, C. N., Heilpern, S., MaldonadoOcampo, J. A., Carvajal-Vallejos, F. M., Encalada, A. C., Rivadeneira, J. F., Hidalgo, M., Cañas, C. M., Ortega, H., Salcedo, N., Maldonado, M. (2018). Tedesco, Fragmentation of Andes-to-Amazon connectivity by hydropower dams. Sci. Adv. 4, eaao1642.

Arauho, C \& Wang, J. (2014). The dammed river dolphins of Brazil: impacts and conservation. Fauna \& Flora International, Oryx. 49 (1): 17-24. Doi: 10.1017/S0030605314000362

Baum, J.K. \& Worm, B. (2009). Cascading top-down effects of changing oceanic predator abundances. J. Anim. Ecol. 78: 699-714.

Buckland, S.T., D.R. Anderson, Burnham, K.P., Laake, J.L. (2001). Introduction to Distance sampling: Estimating abundance of biological populations. Oxford University Press, New York, NY.

Caballero, S., Trujillo, F., Vianna, J.A., Barrios-Garrido, H., Montiel, M.G., Beltran-Pedreros, S., Marmontel, M., Santos, M.C.O., Rossi-Santos, M.R., Santos, F.R., Baker, C.S. (2007). Taxonomic status of the genus Sotalia: Species level ranking for "tucuxi" (Sotalia fluviatilis) and "costero" (Sotalia guianensis) dolphins. Marine Mammal Science. 23 (2): 358-386.

da Silva, V., Trujillo, F., Martin, A., Zerbini, A.N., Crespo, E., Aliaga-Rossel, E. \& Reeves, R. (2018). Inia geoffrensis. The IUCN Red List of Threatened Species 2018: e.T10831A50358152. http://dx.doi.org/10.2305/IUCN. UK.2018-2.RLTS.T10831A50358152.en

Environmental Systems Research Institute (ESRI). (2014). ArcGIS Desktop: Release 10.2.x. Environmental Systems Research Institute, Redlands, CA.

Forsberg, B.R., Melack, J.M., Dunne, T., Barthem, R.B., Goulding, M., Paiva, R.C.D., et al. (2017). The potential impact of new Andean dams on Amazon fluvial ecosystems. PLoS ONE 12(8): e0182254. Doi: 10.1371/journal. pone. 0182254

Gómez-Salazar, C., Trujillo, F., Whitehead, H. (2011). Population size estimates of pink river dolphins (Inia geoffrensis) using mark-recapture methods on photo-identification. Latin American Journal of Aquatic Mammals. 9 (6): 40-47.

Gómez, C., Trujillo, F., Portocarrero-Aya., Whitehead H. (2012). Population, density estimates, and conservation of river dolphins (Inia and Sotalia) in the Amazon and Orinoco river basins. Marine Mammal Science. 28 (1): 124-153.

Gómez-Salazar C., Coll, M., Whitehead, H. (2012a). River dolphins as indicators of ecosystem degradation in large tropical rivers. Ecol.1 Ind. 23 (1):19-26.

Gravena, W., da Silva V.M.F., da Silva M.N.F., Farias, I.P., Hrbek, T. (2015). Living between rapids: Genetic structure and hybridization in botos (Cetacea: Iniidae: Inia spp.) of the Madeira River, Brazil. Biological Journal of the Linnean Society. 114 (4-1): 764-777. Doi: 10.1111/bij.12463

Hrbek, T., da Silva, V.M.F., Dutra, N., Gravena, W., Martin, A.R., Farias, I.P. (2014). A new species of river dolphin from Brazil or: how little do we know our biodiversity. PLoS ONE. 9 (1): e83623. Doi: 10.1371/journal.pone.0083623 
International Whaling Commission (IWC). (2000). Report of the Scientific Sub-Committee on Small Cetaceans. International Whaling Commission Scientific Committee. Journal of Cetacean Research and Management. Supplement 3: 263-291.

Latrubesse, E.M., Arima, E.Y., Dunne, T., Park, E., Baker, V.R., d'Horta, F.M., Wight, C., Wittmann, F., Zuanon, J., Baker, P.A., Ribas, C.C., Norgaard, R.B., Filizola, N., Ansar, A., Flyvbjerg, B., Stevaux, J.C. (2017). Damming the rivers of the Amazon basin. Nature. 546 (7658): 363-369.

Martin, A. R., \& da Silva, V. M. F. (2004). River dolphins and flooded forest: Seasonal hábitat use and sexual segregation of botos (Inia geoffrensis) in an extreme cetacean environment. Journal of Zoology. 263: 295-305.

Mosquera-Guerra F., F. Trujillo., Díaz-Granados, M.C. y H. Mantilla Meluk. (2015). Conservación de delfines de río (Inia geoffrensis y Sotalia fluviatilis) en los ecosistemas acuáticos de la Amazonia y Orinoquia en Colombia. Momentos de Ciencia. 12 (2): 77-86. ISSN 1692-5491.

Mosquera-Guerra F., Parra, C., Trujillo, F., Jiménez-Ortega, A.M., Mantilla-Meluk, H. (2015a). Valoración estacional de las amenazas contra la conservación de Inia geoffrensis humboldltiana (Cetartiodactyla Iniidae) en la cuenca del río Meta, Colombia. Therya. 6 (2): 371-388.

Mosquera-Guerra F., Trujillo, F., Caicedo-Herrera, D., MartínezCallejas, S. (2015b). Indicios de biomagnificación de mercurio total $(\mathrm{Hg})$ en las especies del género Inia (Cetartiodactyla: Iniidae) en los ríos Amazonas y Orinoco (Colombia). Momentos de Ciencia. 12 (2): 145-149.

Mosquera-Guerra, F., Trujillo, F., Caicedo-Herrera, D., ZoqueCancelado, J., Mantilla Meluk, H. (2015c). Impactos de las pesquerías de Calophysus macropterus un riesgo para la salud pública y la conservación de los delfines de río en Colombia. Momentos de Ciencia. 12 (2): 88-99. ISSN $1692-5491$.

Mosquera-Guerra, F., Trujillo, F., Díaz-Granados, M.C., Mantilla-Meluk, H. (2015d). Estimación poblacional y densidad para Inia geoffrensis y Sotalia fluviatilis en los ecosistemas acuáticos de la Amazonia y Orinoquia. Momentos de Ciencia. 12 (2): 93-99. ISSN 1692-5491.

Mosquera-Guerra, F., Trujillo, F., Danni, P., Oliveira-da-Costa, M., Marmontel, M., Armenteras-Pascual, D., Usma, S., Willems, D., Carvajal-Castro, J.D., Mantilla- Meluk, H., Franco, N., Amorocho, D., Maldonado, R., Berg, K., Sainz, L., Van Damme, P.A., Cambell, E. (2018). Analysis of distribution of river dolphins (Inia and Sotalia) in protected and transformed areas in the Amazon and Orinoco basins. SC/67B/SM/16. Bled, Slovenia.

Mosquera-Guerra, F., Trujillo, F., Parks, D., Oliveira-da-Costa, M., Usma, S., Willems, D., Maldonado, R., Amorocho, D., Berg, K., Armenteras-Pascual, D., Van Damme, P.A., Sainz, L., Franco, N., Mantilla-Meluk, H., CarvajalCastro, J.D., Cambell, E., Cordova, L., Echeverria, A., Caballero, S., Marmontel, M. (2018a). Presence of mercury in river dolphins (Inia and Sotalia) in the Amazon and Orinoco basins: evidence of a growing threat for these species. SC/67B/SM/16. Bled, Slovenia.

Mosquera-Guerra, F., Trujillo, F., Oliveira-da-Costa, M., Marmontel, M., Armenteras- Pascual, D., Usma, S., Willems, D., Carvajal-Castro, J.D., Mantilla-Meluk, H., Franco, N., Amorocho, D., Maldonado, R., Berg, K., Sainz, L., van Damme, P.A. (2018b). Movements and habitat use of river dolphins (Cetartiodactyla: Iniidae) in the Amazon and Orinoco river basins, determined from satellite tagging. SC/67B/SM/16. Bled, Slovenia.

Pavanato, H., Melo-Santos, G., Lima, D., Portocarrero-Aya, M., Paschoalini, M., Mosquera, F., Trujillo, F., Meneses, R., Marmontel, M., Maretti, C. (2016). Risk of dam construction for South American River Dolphins: a case of study of the Tapajós River. Endangered Species Research. 31: 47-60. Doi: 10.3354/esr00751.

Perrin, W. F., Brownell, R. L., Zhou, K., Jiankang, L. (1989). Biology and conservation of the river dolphins. Proceedings of the Workshop on Biology and Conservation of the Platanistoid Dolphin held at Wuhan, People's Republic of China, October 28-30, 1986. Occasional Papers of the IUCN species survival commission (SSC). 3: 173.

Pires, A. (2006). Community timber enterprises in the Mamiraua Sustainable Development Reserve, Brazil. Communitybased forest enterprises in tropical forest countries: Scoping study: 27. Instituto de Desenvolvimento Sustenta 'vel Mamiraua', Brazil. Fecha de consulta: 22 de septiembre, 2018. Disponible en: http://www.rightsandresources.org/ documents/files/doc_295.pdf

Reeves, R. R. \& Leatherwood, S. (1994). Dolphins, Porpoises and Whales 1994-1998 Action Plan for the Conservation of Cetaceans. IUCN Species Survival Commission, Gland, Switzerland.

Reeves, R. \& Leatherwood, S. (1994a). Dams and river dolphins: can they co-exist. Royal Swedish Academy of Sciences. 23 (3): $172-175$.

Rosas, F.C.W., \& Kesä, K.L. (1996). Nutritional and mercury content of milk of the Amazon river dolphin, Inia geoffrensis. Comp.Biochem. Physiol. 115A (2): 117-119.

Sanderson, E.W., Jaiteh, M., Levy, M.A., Redford, K.H., Wannebo, A.V., Woolmer, G. (2002). The human footprint and the last of the wild. Bioscience. 52: 891-904.

Sandin, S.A., Smith, J.E., DeMartini, E.E., Dinsdale, E.A., Donner, S.D., Friedlander, A.M., Konotchick, T., Malay, M., Maragos, J.E., Obura, D. (2008). Baselines and degradation of coral reefs in the northern Line Islands. PLoS ONE. 3: 1-11.

Sergio, F., Caro, T., Brown, D., Clucas, B., Hunter, J., Ketchum, J., McHugh, K., Hiraldo, F. (2008). Top predators as conservation tools: ecological rationale, assumptions, and efficacy. Annu. Rev. Ecol. Evol. Syst. 39: 1-19.

Sioli, H. (1984). The Amazon and its main affluents: Hydrography, morphology of the river courses, and river types. p. 127166. In The Amazon, Limnology and landscape ecology of a mighty tropical river and its basin. H. Sioli, editor. Dr. W. Junk Publishers. The Hague, The Netherlands.

Shostell, J.M \& Ruiz-García, M. (2010). An introduction to river dolphin species. In: Biology, Evolution and Conservation of River Dolphins. Editors: Manuel Ruiz-García and Joseph Shostell. Nova Science Publishers, Inc. New York. p.1-28.

Smith, B., Braulik, G., Strindberg, S., Ahmed, B., Mansur, R. (2006). Abundance of Irrawaddy dolphins (Orcaella brevirostris) and Ganges river dolphins (Platanista gangetica gangetica) estimated using concurrent counts made by independent teams in waterways of the Sundarbans mangrove forest in Bangladesh. Marine Mammal Science. 22: 527-5477s 
Platanista gangetica. In: IUCN 2008. IUCN Red List of Threatened Species. Conservation of Indus River Dolphin (Platanista gangetica minor) in the Indus River System, Pakistan: an overview | Request PDF. Fecha de consulta: 14 de diciembre, 2008. Disponible en: https://www. researchgate.net/publication/259240004_Conservation of_Indus_River_Dolphin_Platanista_gangetica_minor_in_the_Indus_River_System_Pakistan_an_overview [accessed Sep 17 2018]

Thomas, L., B S.T. Buckland, E.A. Rexstad, J.L. Laake, S. Strindberg, S. Hedley, J.R.B. Bishop, Marques, T., Burnham, K.P. (2010). Distance software: Design and analysis of distance sampling surveys for estimating population size. Journal of Applied Ecology. 47: 5-14.

Turvey, S.T., Pitman, R.L., Taylor B.L., Barlow, J., Akamatsu, T., Barrett, L.A. Zhao, X., Reeves, R.R., Stewart, B.S., Wang, K., Wei, Z., Zhang, X., Pusser, L. T., Richlen, M., Brando, J.R., Wang, D. (2007). First human-caused extinction of a cetacean species? Biology letters. The Royal Society. 3: 537-540.

Trujillo, F. (2000). Habitat use and social behaviour of the freshwater dolphin Inia geoffrensis (de Blainville 1817) in the Amazon and Orinoco basins. Ph.D. thesis, University of Aberdeen, Aberdeen, Scotland. 157 p.

Trujillo, F., Crespo, E., Van Damme, P., Susma, J. (Eds). (2010). The Action Plan for South American River Dolphins 20102020. WWF, Fundación Omacha, WCS, WDCS, Solamac. $240 \mathrm{p}$.

Trujillo, F., Crespo, E., Van Damme, P.,Usma, S., MoralesBetancourt, D., Wood, A., Portocarrero, M. (2010a). Summary of threats for river dolphins in South America: past, present and future. p. 145-158. In: Trujillo, F., Crespo, E., Van Damme, P. \& J. Susma (Editores). The Action Plan for South American River Dolphins 2010-2020. WWF, Fundación Omacha, WCS, WDCS, Solamac. 240 p.
Trujillo, F., Gómez-Salazar, C., Portocarrero, M., AliagaRossel, E., Utreras, V., Usma, S., Tavera, G. (2011). Estimaciones de abundancia de delfínes de río: una herramienta para diseñar estrategias de conservación. Pp. 57-70. En: Trujillo, F., Crespo, E., van Damme, P., Usma, J. (2011). Plan de Acción para la conservación de los delfines de río en Sudamérica: Resumen Ejecutivo y Avances 2010-2020. WWF, WCS, WFN, Solamac, Fundación Omacha, 104 p.

Trujillo, F., C. Gómez., S. Martínez., Herrera, O., MosqueraGuerra, F. (2016). Estimaciones de densidad, y conservación del delfín de río (Inia geoffrensis) en el río Meta. Pp 2742 a 28. En: T rujillo, F; R. Antelo \& Usma S. (editores). 2016 Biodiversidad de la cuenca baja y media del río Meta. F undación Omacha, Fundación Palmarito, WW F. Bogotá 336 páginas.

US Geological Service. (2018). HydroSHEDS. Fecha de Consulta: 14 de diciembre, 2008. Disponible en: https://hydrosheds. cr.usgs.gov/

Vidal, O., Barlow, J., Hurtado, L., Torre, J., Cendon, P., Zully, O. (1997). Distribution and Abundance of the Amazon River Dolphin (Inia geoffrensis) and the Tucuxi (Sotalia fluviatilis) in the upper Amazon. Mar. Mamm. Sci. 13 (3): 427-445.

Utreras, V., Trujillo, F. Usma, S. 2013. Plan de Acción para la Conservación de los mamíferos Acuáticos de Ecuador. Ministerio de Ambiente de Ecuador, WWF, WCS, Fundación Omacha. 124 p.

Williams, R., Moore, J., Gomez-Salazar, C. Trujillo, F., Burt, L. (2016). Searching for trends in river dolphin abundance: Designing surveys for looming threats, and evidence for opposing trends of two species in the Colombian Amazon. Biological Conservation. 195: 136-145. 